\title{
Enquête sur le « suicide » de Victor Bach
}

\section{Bernard Gainot}

\section{(2) OpenEdition}

Journals

Édition électronique

URL : https://journals.openedition.org/ahrf/288

DOI : 10.4000/ahrf.288

ISSN : $1952-403 X$

Éditeur :

Armand Colin, Société des études robespierristes

Édition imprimée

Date de publication : 1 décembre 1999

Pagination : 615-637

ISSN : 0003-4436

\section{Référence électronique}

Bernard Gainot, «Enquête sur le « suicide » de Victor Bach », Annales historiques de la Révolution française [En ligne], 318 | octobre-décembre 1999, mis en ligne le 11 avril 2006, consulté le 24 avril 2022. URL : http://journals.openedition.org/ahrf/288 ; DOI : https://doi.org/10.4000/ahrf.288

Ce document a été généré automatiquement le 24 avril 2022.

Tous droits réservés 


\title{
Enquête sur le « suicide » de Victor Bach
}

\author{
Bernard Gainot
}

À François Hincker

1 Les historiens de la "révolution de brumaire » insistent généralement sur le caractère pacifique de ce changement de régime, qui ne provoqua la mort de personne, au cours de laquelle aucun coup de feu ne fut tiré, et où même les listes de proscription n'eurent pour effet immédiat que des tracasseries mineures à l'égard de ceux qui y furent portés.

2 Nous n'entrerons pas dans la discussion sur les causes de cette " révolution pacifique ", ni sur les interprétations fort contradictoires que donnèrent à chaud les contemporains de l'événement ${ }^{1}$. Nous repartirons plutôt des représentations : celles qui sont les plus couramment admises enferment l'événement dans un espace clos (l'orangerie du château de Saint-Cloud), marquant par là même la phase terminale de la Révolution (les «masses» ont été refoulées de l'espace politique), dramatisant l'affrontement des ultimes défenseurs du système représentatif, les représentants en toge du Conseil des Cinq-Cents, et des grenadiers protégeant un Bonaparte silencieux et mal à l'aise d'une haie de baïonnettes plus ou moins menaçantes ${ }^{2}$. Cette représentation s'impose, et se fixe au milieu du XIX siècle autour du tableau de François Bouchot, accordant aux défenseurs du système représentatif une part plus honorable que dans les gravures contemporaines de l'événement ${ }^{3}$.

3 Une exception pourtant dans cette représentation conventionnelle de la " révolution de brumaire ", le tableau de J. Le Cœur au musée Carnavalet, intitulé : La mort deVictor Bach le 18 Brumaire, au pied de la statue de la Liberté, place de la Concorde. L'œuvre n'est pas datée, mais son inspiration « républicaine » ne fait aucun doute, à tel point qu'elle a pu figurer dans une exposition récente des Archives nationales commémorant le bicentenaire de la République ${ }^{4}$. Si le décalage avec les représentations conventionnelles (au niveau du lieu, et bien sûr du thème central) est patent, ce tableau ne contredit pas pour autant l'historiographie dominante: la mort solitaire du démocrate, en un lieu « hanté » par les grandes journées révolutionnaires, prouve que la révolution est terminée. Mort pathétique, laissant totalement indifférents les 
travailleurs urbains et les militants républicains qu'elle est censée interpeller. En fait, si nous ne savons rien de son auteur, la facture du tableau nous permet de le situer dans les années $1880^{5}$.

4 L'accumulation de détails précis, jusqu'à ce papier à côté de la tête du cadavre, daté du 18 brumaire an VIII, sans autre contenu que le mot France, accentue l'effet positif; il ne doit pas faire de doute pour le spectateur qu'un fait se soit bien produit ici même, au moment où la grande histoire semble se faire ailleurs, et que le caractère dramatique de ce fait contredit l'historiographie dominante : la «révolution de brumaire » a fait au moins une victime... Mais quel est donc ce cadavre?

5 La notice du catalogue de l'exposition synthétise les maigres renseignements dont disposait alors l'auteur à l'époque où il réalisa ce tableau : «Victor Bach, médecin né à Villefranche-de-Rouergue vers 1770, participe à la Conjuration des Égaux de Gracchus Babeuf, désespéré par l'établissement d'une dictature militaire, le 18 brumaire, il se suicide au pied de la statue de la Liberté, place de la Révolution. »

6 En peu de mots, tout est dit: la jeunesse (Bach atteindrait tout juste la trentaine), le radicalisme (allusion au babouvisme), le lieu précis et la motivation de l'acte (contexte politique). Les notices du XIX siècle tournent toutes autour de ces quelques touches qui campent un portrait.

7 Commençons par la première de toutes, celle de la Biographie des Contemporains (1821) ${ }^{6}$. Elle sort de l'ombre la personnalité de Bach, tout en figeant son image pour la postérité. C'est pourquoi il faut la citer dans son intégralité :

«Médecin de Paris, électeur du département de la Seine en l'an VI (1798) ; l'un des partisans les plus enthousiastes et les plus exaltés de la Révolution. Il fut arrêté par ordre du gouvernement directorial, et traduit devant un jury d'accusation, comme auteur d'un écrit satirique contre les membres du Directoire, et plus particulièrement contre les auteurs de la loi du 22floréal an VII (11 mai 1799) [sic] ${ }^{7}$ qui annulait une partie des élections des collèges électoraux.

Mis en liberté, Bach n'en continua pas moins d'attaquer avec véhémence les directeurs et tous les ennemis de la Révolution. En juin 1799 (prairial an VII), il prononça à la tribune des jacobins, rue du $\mathrm{Bac}^{8}$, un discours très violent, dans lequel il s'élevait de nouveau contre le Directoire, exposait les dangers qui menaçaient la Patrie, et proposait la création d'un gouvernement exclusivement démocratique. Il termina ce discours par la lecture d'un projet de constitution, où il donnait à la démocratie une telle extension, que la constitution de 1793, surnommée le Code de l'anarchie, n'aurait été qu'une œuvre aristocratique.

La révolution du 18 brumaire an VIII, et l'élévation du général Bonaparte au Consulat troublèrent sa raison déjà fort ébranlée; il se donna la mort au pied de la statue de la Liberté, qui avait remplacé celle de LouisXV, sur la place de la Révolution.»

Voici donc exposés les traits marquants ; le médecin, le terroriste, l'ultra-démocrate, le suicide clairement localisé et contextualisé (une conséquence de la « révolution de brumaire an VIII »). Dans la première édition du Dictionnaire biographique de Michaud ', en 1811, Victor Bach est ignoré ; en revanche, ce dernier apparaît dans le Supplément de 1834 , avec une notice rédigée par Michaud jeune ${ }^{10}$. Le personnage est présenté avec un a priori hostile ; « révolutionnaire fanatique ", lié avec tout ce qu'on trouve à Paris de "démagogues et d'anarchistes turbulents ". Mais Michaud apporte tout de même d'utiles précisions complémentaires: sur la jeunesse de Bach («né vers 1770 à Villefranche - Aveyron, d'une famille de cultivateurs-propriétaires ») et sur la date de son arrivée à Paris ( Il alla vers le commencement de 1790 achever ses cours [de 
médecine] à Paris»). C'est également dans cette notice que nous trouvons le récit le plus détaillé de la mort du médecin démocrate :

« Il alla un matin se prosterner devant la statue de la Liberté, qui existait encore sur la place Louis-XV, dans l'endroit même où la tête de Louis XVI était tombée ; et là, maudissant la tyrannie qui pesait sur la France, il se brûla la cervelle d'un coup de pistolet. Ce fait remarquable, et qui prouve au moins que Bach était un républicain de conviction, fit très peu de bruit, la police consulaire, dès lors très vigilante, ayant tout fait pour l'étouffer. »

C'est la seule fois que nous trouvons mentionnée cette intervention de la police, qui pose un certain nombre de questions sur la construction du récit.

Enfin, Michaud inscrit nettement l'action politique de Bach dans le cadre du babouvisme et du néo-babouvisme : "Après la chute de Robespierre, il fut à son tour persécuté, et il n'échappa qu'avec peine aux poursuites qui furent alors dirigées contre les complices de Babeuf et les agresseurs du camp de Grenelle ». Il semble ici qu'il y ait une certaine confusion entre la répression du mouvement démocratique en l'an III, et celle de l'année suivante (an IV) qui dessine le contexte de la conjuration des Égaux. Quant au discours du Club du Manège, il ne fait aucun doute non plus pour Michaud que son objet était de développer un "projet de constitution » qui place Victor Bach parmi les héritiers du «Tribun » : « Il demanda sans détour la loi agraire, le partage des biens, et proposa sans détour un projet de constitution dont les bases étaient plus démocratiques encore que celles qui avaient été présentées par Robespierre cinq ans auparavant. »

11 La Nouvelle Biographie Universelle, du milieu du xixe siècle, accentue encore, sans apporter de preuves supplémentaires, la parenté idéologique entre le prétendu projet de constitution présenté au Manège et le néo-babouvisme : « Il parla au Club du Manège sur les dangers de la Patrie, et proposa, pour la sauver, une contitution dont les idées se rapprochaient du système Babeuf. " ${ }^{11}$

12 Telles sont les traces indirectes que Le Cœur avait à sa disposition lorsqu'il prit Bach pour modèle d'une résistance impuissante au coup d'État militaire.

Depuis, la connaissance du personnage historique reste un peu évanescente; dans la dernière notice biographique qui lui est consacrée ${ }^{12}$, datant de l'immédiat aprèsguerre, Gérard Walter fait le point à partir des quelques sources écrites et des deux imprimés revendiqués par Victor Bach ${ }^{13}$. C'est la notice biographique la plus complète que nous ayons à ce jour ; nous verrons qu'il est possible de la préciser ou de la corriger sur certains points.François Hincker m'avait fortement encouragé à en savoir un peu plus sur ce personnage énigmatique, ayant laissé fort peu de traces de sa courte existence, véritable "énergumène " pour les uns, témoin lucide de l'assassinat de la République pour les autres. Il m'avait notamment indiqué un numéro spécial du Bulletin de la Société des Amis de Villefranche et du Bas-Rouergue, dans lequel un article faisait le point sur les origines provinciales de Bach ${ }^{14}$. C'est la raison pour laquelle cet article est dédié à la mémoire de mon ex-collègue.

14 Le «cas Bach» nous offre l'occasion de réévaluer la connaissance positive que nous avons du mouvement démocratique sous le Directoire et le Consulat, telle qu'elle transparaît dans les notices biographiques présentées en préalable. Car nous pensons d'abord que les faits ne sont pas clairement établis : le rapport entre le "suicide » de Bach et la «révolution de brumaire », ou la nature du « radicalisme » qui transparaît dans ses écrits, pour ne citer que des faits qui paraissent s'imposer par leur évidence. 
Ensuite, cet exemple nous permet d'exposer d'autres approches possibles, et de formuler des hypothèses; sur la mise en réseaux, ou sur les rapports entre connaissance positive et représentations, par exemple.

La première des interrogations naît de la méditation sur le tableau de Le Cœur ; l'artiste a joué sur deux registres. Le registre symbolique relève du divorce entre République et Révolution: les signes républicains sont visibles (les drapeaux tricolores sur le ministère de la Marine et les Tuileries, l'inscription sur le socle de la statue), mais ne servent plus qu'à planter le décor d'une scène quasiment vide, soulignant le dramatique isolement du jeune mort du premier plan. Le registre positif, nous l'avons déjà noté, multiplie les référents temporels; papier daté, costume, brouillard. Ce second registre, lourdement appuyé, n'est qu'une instance de légitimation du registre symbolique; il fait passer pour vraie une réalité entièrement recomposée par le peintre, à partir des traces écrites qu'il avait à sa disposition.

Le cadavre exposé est celui d'un homme jeune, voire très jeune, vêtu d'une tenue soignée évoquant vaguement le costume de ville d'un représentant du peuple (on devine une écharpe tricolore autour de la taille). Il s'est effondré sur le socle de l'ancienne statue de Louis XV, démontée et remplacée par une statue de la Liberté, qui semble encore avoir fière allure sur le tableau, alors que, d'après les journaux de l'époque, cette effigie en plâtre menaçait ruine au début du Consulat. Elle devait être rapidement et brièvement démolie, sans que cela soulève d'émotion particulière ${ }^{15}$.

Quant au fait lui-même, il doit logiquement avoir laissé une trace, soit par identification du cadavre, soit par un récit dans les journaux de l'époque. L'identification des individus disparus à la suite d'une mort violente se fait grâce aux registres de la basse geôle de l'ancien Châtelet de Paris. Richard Cobb a présenté cette source et les multiples voies offertes à l'historien pour son exploitation ${ }^{16}$.

18 La consultation de ces registres n'offre pourtant qu'une seule mention d'un suicide place de la Concorde (tel est le nom de l'ancienne place de la Révolution depuis 1795) dans des circonstances identiques à celles du prétendu suicide de Victor Bach; un individu de sexe masculin s'est «brûlé la cervelle », à la date du 3 frimaire an VIII (24 novembre 1799), donc une quinzaine de jours après le coup d'État ${ }^{17}$. Mais cet individu a pu être identifié ; il s'appelait Carré, et son acte a été suffisamment remarqué pour que Le Moniteur nous relate longuement les circonstances du suicide :

« Le 3 de ce mois, à dix heures du soir, un citoyen s'est brûlé la cervelle sur la place de la Révolution, aux pieds de la Statue de la Liberté. Il serait possible qu'on attribuât ce suicide à une cause peu étrangère aux derniers événements; on a répandu le bruit que le suicidé était un des inscrits sur la liste des déportés; d'autres ont cru voir dans cet acte de désespoir une imitation de la fin tragique de Caton, ne voulant pas survivre à la perte de la Liberté ; d'autres encore ont pensé que, victime des malheurs de la révolution, l'infortuné avait choisi cette place pour y accuser en mourant et la révolution et ses principes. Ainsi, chacun a jugé cet événement suivant ses vues personnelles; mais il est certain que le suicide ne tenait à aucune cause extraordinaire. Le citoyen Carré, du département du Loiret (c'est le nom de l'individu) avait joui longtemps d'une aisance dont il avait contracté l'habitude plus que prévu le terme. L'aspect du besoin, prêt à l'assiéger, lui devient insupportable. Il comptait sur des protecteurs puissants; ses espérances trompées achevèrent d'égarer sa tête; déjà on avait pu y apercevoir quelque signe de dérangement. Quelque temps avant sa mort, passant sur la place de la Révolution avec sa femme et un ami, il leur dit froidement que ce lieu lui paraissait commode pour terminer sa vie, et qu'il le choisirait si jamais il prenait cette fatale résolution. Il a tenu malheureusement parole. Une lettre adressée à sa femme, qu'il chérissait 
tendrement, a indiqué le lieu où l'on devrait le trouver, et où l'on a reconnu ses restes. » ${ }^{18}$ différentes notices sur la mort de Bach : la confusion serait donc possible avec ce Carré, dès cette époque, puisque le journal mentionne la rumeur : «On a répandu le bruit que le suicidé était un des inscrits sur la liste des déportés ». Or, Victor Bach a bien été inscrit sur les listes de proscription, le 20 brumaire an VIII.Quant à l'« imitation de la fin tragique de Caton ", nous verrons que les figures du "suicide héroïque à l'antique ", hantent littéralement les rares pièces imprimées que nous ait laissées le médecin démocrate. Se peut-il qu'une mémoire jacobine ait volontairement entretenu l'équivoque entre le suicide, pour des mobiles d'ordre purement privé, de ce Carré, et celui de Bach? Ou bien l'équivoque est-elle délibérément entretenue par la police, qui aurait ainsi fait disparaitre et le cadavre et la trace d'une protestation politique ? Peutêtre est-ce le fait sous-entendu par la notice, par ailleurs plutôt bien informée, de Michaud? Mais ce serait attribuer à la «police du Consulat » une redoutable efficacité, dès ses origines: l'article cité du Moniteur attirait déjà notre attention sur le choix délibéré du lieu de son suicide par Carré, cette mise en scène qui introduit la confusion entre la publicité et le mobile privé. L'auteur dudit article y voit un «signe de dérangement " de l'esprit du futur suicidé. Rappelons-nous que certaines notices mentionnaient également le fait que la raison de Bach avait été profondément perturbée par la nouvelle du coup d'État. Mais comment croire que, si le suicide en pleine nuit d'un obscur citoyen a pu faire l'objet d'un long article, celui d'un personnage aussi connu de l'opinion parisienne que l'était alors Victor Bach, n'ait pu laisser aucune trace dans la presse de l'époque, par ailleurs encore relativement libre ? aurait-il mis fin à ses jours?

Peu après l'affaire de la machine infernale (attentat du 3 nivôse an IX), le ministère de la Police opère une rafle dans les milieux démocratiques de la capitale; Bach est de nouveau porté sur les listes de personnes à déporter dix jours plus tard, le 13 nivôse an IX (3 janvier 1801). Mais un rapport de police corrige aussitôt en face de son nom: "s'est tué au bois de Boulogne d'un coup de pistolet, il y a six mois » ${ }^{19}$. Ce qui placerait donc le suicide de Bach fin juin-début juillet 1800 (début messidor an VIII), dans le contexte assez troublé de la crise de Marengo.

Après nouvelle consultation des registres de la basse geôle, nous trouvons bien la mention d'un suicide dans le bois de Boulogne le 16 prairial anVIII (5 juin 1800), mais le cadavre a pu être identifié ; il s'agit d'un certain Arson ${ }^{20}$.

L'une des clefs de l'énigme du "suicide» de Victor Bach réside dans le fait qu'il ne s'agit pas d'un inconnu, mais d'une personnalité de premier plan de l'opposition démocratique au Directoire. Cette notoriété doit maintenant retenir notre attention, puisqu'elle lui vaut la réputation de «radicalisme », d'» anarchisme », d'» ultradémocratisme ». Cette réputation est essentiellement fondée sur l'un de ses écrits, le Discours qu'il prononça et fit imprimer le 30messidor an VII (18 juillet 1799) à la tribune du Club du Manège. Il y aurait exposé un "système" dérivé de celui de Babeuf, et présenté un « projet de constitution » ultra-démocratique ${ }^{21}$.

En réalité, lorsque Bach prend la parole en cet été 1799 pendant quarante-cinq minutes, ce n'est certes pas un inconnu pour l'assistance qui se presse dans la salle du jardin des Tuileries.L'année précédente, il avait été placé au cœur des controverses électorales ; 
président de l'une des trois assemblées primaires de la division des Invalides (Xe arrondissement), il fut choisi comme électeur, puis comme scrutateur de l'un des bureaux de l'assemblée électorale de la Seine séant à l'Oratoire, présidé par Antonelle ${ }^{22}$. C'est ce choix qui est l'un des principaux motifs de la scission de l'assemblée électorale, dont les péripéties et les conséquences ont été analysées par ailleurs ${ }^{23}$.

Le pamphlet autojustificatif que fait alors imprimer Victor Bach est accompagné des quelques pièces qui nous permettent de situer un peu mieux le rang social du personnage.Plusieurs électeurs conservateurs font valoir qu'il ne remplit pas les conditions nécessaires pour remplir des fonctions politiques, puisqu'il n'atteint pas le seuil de revenus : selon eux, Bach aurait même adopté une attitude provocatrice face aux normes censitaires, en se vantant de ne pas payer de contributions.

Les documents qu'il produit en réponse à ses détracteurs montrent que les sources de ses revenus déclarés sont essentiellement en dehors du département de la Seine. Il est né à Villefranche-de-Rouergue en 1764, dans une famille de forgerons. Il a donc trentecinq ans au moment de sa mort, c'est un homme mûr ; alors que le tableau de Le Cœur, qui suit les premières indications biographiques, représente un jeune homme.

Il est le troisième d'une famille de sept enfants, plutôt acquise aux idées nouvelles. Son père Jean Bach est franc-maçon, son cousin Pierre Bach joue un rôle important sur le plan local à l'époque de la Terreur. Tout laisse à penser que le médecin parisien a gardé des contacts étroits avec son milieu familial d'origine; son oncle lui a servi d'intermédiaire pour obtenir de la municipalité de Villefranche une attestation portant sur des biens-fonds, évalués à plus de deux cents journées de travail. Et ce parent ajoute dans sa lettre : «Lorsqu'on a, comme toi, des propriétés pour mille journées de travail, il n'est pas difficile d'avoir des attestations pour deux cents journées ». Bach remplirait donc largement les conditions censitaires imposées au deuxième degré par la Constitution de l'an III ${ }^{24}$.

28 Il a fait ses études au collège de Villefranche, puis il est parti pour Montpellier, en 1786, où il a été reçu chirurgien. Dans la suite de sa carrière, il tient à faire suivre sa signature de son titre de "médecin » (ou même "docteur en médecine de l'École de Montpellier »). Selon ses biographes, c'est en fait pour achever son cursus à l'École de Médecine qu'il s'installe à Paris en février $1790{ }^{25}$. Mais il n'aurait jamais exercé la médecine, et se serait tout de suite lancé dans la politique; affirmation difficile à vérifier, puisque la pratique professionnelle en ce cas n'était pas soumise à imposition. Nous n'avons par ailleurs pas trouvé d'indications quant à son rattachement à une institution hospitalière.

29 Certes, on peut penser que ce premier degré de qualification, acquis dans une université prestigieuse, est un laissez-passer fort utile, et d'ailleurs fièrement revendiqué, pour un représentant de la petite bourgeoisie des «talents », pour un jeune provincial qui fait ses premières armes à Paris. Mais pourquoi conserve-t-il si manifestement cette référence par la suite? Les propriétés foncières d'une famille artisanale aisée, mais nombreuse, suffisaient-elles à lui garantir un statut de "petit rentier»? Le seuil des mille francs annuels de revenus autorise cette hypothèse; l'étiquette professionnelle servirait alors de marqueur d'identité sociale.

30 Très engagé dans le mouvement révolutionnaire, des documents de l'anIII le mentionnent comme "ancien commissaire de police» de la section des Thermes (ou section Chalier) ${ }^{26}$. Albert Soboul cite également une dénonciation du 20 prairial an III (8 juin 1795) pour des faits qui remontent au printemps précédent; le 20 germinal de 
l'an II, Bach dénonçait les riches de sa section qui avaient offert à une collecte pour le salpêtre une somme inférieure à celle recueillie parmi les ouvriers de l'atelier des poudres ${ }^{27}$. D'autres rapports le présentent comme "un partisan» de Carrier. Ces diverses pièces de la période thermidorienne vont désormais lui assurer une réputation de «terroriste ", de « robespierriste convaincu " ${ }^{28}$.

31 À cette époque, Bach habitait la rue Saint-Jacques. Sous le Directoire, il a changé de domicile : il s'est déplacé vers l'ouest, mais toujours sur la rive gauche. Il est désormais locataire dans la maison de Sens, au faubourg Saint-Germain, à l'extrémité occidentale de la rue de Grenelle ${ }^{29}$. Il y occupe un appartement meublé; les militants démocrates avaient coutume de se retrouver dans ces ensembles locatifs, que les rapports de police de la période directoriale et consulaire nous ont rendus familiers (l'Abbaye-au-bois, l'ex-couvent de l'Annonciation, l'ancien séminaire de Saint-Sulpice, etc.). Il se trouve tout près de l'Hôtel des Invalides, où les néo-jacobins trouvent un nombre élevé de partisans. Il fréquente la taverne du Gros-caillou, dont le propriétaire est l'invalide Gomigeon, qui se fit remarquer à Grenelle, et qui fut de nouveau mis en cause lors des incidents violents qui agitèrent les assemblées primaires de l'an VI. À quelques pas en direction de l'est, on croise la rue du Bac; là, se réunissent à la fin du Directoire, les dirigeants les plus influents de l'opposition démocratique parisienne ; Bach est membre de la Société Politique, où il peut rencontrer Xavier Audoin, Antonelle, Félix Le Peletier, René Vatar, l'adjudant Jorry, etc.

L'activité militante de Bach est donc exempte de solution de continuité depuis la période du gouvernement révolutionnaire; avant cette date, nous n'avons plus de traces écrites. Cette activité fonde la notoriété de Bach, et lui permet d'obtenir les suffrages nécessaires aux élections de l'an VI.

La «floréalisation» qui s'ensuivit élargit encore ses assises dans les réseaux démocratiques de la capitale. Notons qu'il n'a pas coupé pour autant les ponts avec son département d'origine; outre les relations familiales, il rappelle en 1799 qu'il a fait parvenir à un ami du député de l'Aveyron, Cambe, le texte de son second discours ${ }^{30}$; relations qui l'insèrent tout à fait dans les réseaux politiques de la période directoriale.

Ce n'est assurément pas un inconnu, disions-nous, pour les sociétaires du Manège en juillet 1799. Son discours est très chaleureusement accueilli. À l'extérieur, il déclenche de vives polémiques: L'Ami des lois de Poultier l'accuse de prêcher la loi agraire ${ }^{31}$ prétexte à la dénonciation d'une vaste " conspiration jacobine", mise en scène par Sieyès et Lucien Bonaparte. Le discours de Bach, dans la mesure où il illustrerait l'interprétation qu'en ont donné ses détracteurs (la présentation d'un "projet de constitution" qui propose un programme de revendications sociales proche du "système de Babeuf»), serait une véritable provocation. Il fournit aux conservateurs l'occasion tant attendue pour démontrer à la majorité des députés (le Corps législatif, et surtout le Conseil des Anciens, détient les pouvoirs de police sur les bâtiments des Tuileries, dont la salle du Manège) que, contrairement à leurs démonstrations légalistes, les néo-jacobins ne souhaitent que le renversement de la Constitution de l'an III et la suppression de la propriété privée (ou plus exactement un «nivellement » des biens). Brandir ainsi l'épouvantail du " péril rouge » offre, dans un premier temps, une opportunité pour fermer la Salle du Manège, et ruiner la stratégie de l'état-major démocrate regroupé autour de l'équipe du Journal des Hommes libres; stratégie qui vise à réunir les différents réseaux militants de Paris et de province, et les noyaux parlementaires (une soixantaine de députés), à un centre libéral, afin de constituer une 
majorité dans les Conseils susceptible d'aménager progressivement la Constitution de l'an III en « démocratie représentative ».

Le discours de Bach aurait donc permis la fermeture effective de la Salle du Manège le 8 thermidor (26 juillet), et introduit une division fatale au sein du mouvement démocrate. C'est du moins ce que laissent supposer la plupart des notices biographiques et récits insérant le discours dans le cours politique de cet été 1799 .

Je ne pense pas que cette lecture soit la bonne, et que le Premier discours refléterait un clivage politique entre « radicaux » nostalgiques de la Terreur, et « opportunistes » plus attentifs aux alliances pour ménager l'avenir (dont les porte-parole seraient Félix Le Peletier et Antonelle) ${ }^{32}$.

Parmi les traits majeurs du discours, retenons d'emblée qu'il confirme l'appartenance de l'orateur à la bourgeoisie des " talents », et que cette appartenance est ouvertement revendiquée, non seulement par sa qualification professionnelle, ainsi que nous l'avons déjà mentionné, mais aussi par les références culturelles dont ce discours est truffé. Les exemplae tirés de l'Antiquité gréco-romaine sont légion, bien plus fréquents que dans les autres interventions d'orateurs du Club du Manège qui nous sont parvenues. Sont évoqués (pêle-mêle) Léonidas, Mutius-Scaevola, Curtius, mais aussi Sylla, Brutus, Manlius, Torquatus, Phocion, Philomène, etc. Rhétorique souvent bien lourde, plombée par la hantise du "suicide héroïque " ${ }^{33}$, les exemplae qui mettent en scène Léonidas et Curtius évoquent explicitement cette "mort glorieuse", constamment présente à l'esprit de Bach ${ }^{34}$. Il y a là un indice plus probant de la fin supposée du médecin démocrate, que tout ce que contiennent les registres des justices de paix ou de la police.

L'évocation du passé est fréquemment présente, mais elle n'est pas une pure et simple nostalgie du retour à 1793. Certes, les épisodes tragiques qui jalonnent l'expérience démocratique, sont évoqués comme autant de "leçons " ${ }^{35}$ dont la mémoire entretenue doit servir à forger la maturité du mouvement. Un autre élément indispensable à la constitution de cette mémoire collective est le processus d'héroïsation des victimes de l'oligarchie ; sont donc convoqués les mânes des martyrs de prairial, ceux de Grenelle et ceux de Vendôme. Cette évocation est le passage le plus souvent cité du discours de Bach, et cette fréquence entretient l'équivoque sur la nature nostalgique de la " résurgence » jacobine de $1799{ }^{36}$. Toutefois, ce «culte des martyrs " n'est pas une originalité qui suffirait à elle seule à fonder la radicalité du discours de Bach, par rapport au courant néo-jacobin converti à l'opportunisme. Isser Woloch nous rappelle que la convocation des mânes des ancêtres sacrifiés pour la cause est devenue un "topos" de la rhétorique néo-jacobine ${ }^{37}$ ayant pour fonction de constituer et de renforcer l'identité collective. L'originalité réside plutôt dans le style: sont non seulement invoquées les figures emblématiques des martyrs (prairial-VendômeGrenelle), mais également l'ensemble des victimes de l'oligarchie, tant en France que dans les républiques sœurs. Cette procession de fantômes qui réclame justice est évoquée avec des accents préromantiques, en un tableau saisissant qui est une réminiscence des scènes du Jugement Dernier, qui ornaient le tympan des églises :

«... calculez, si vous le pouvez, la somme des vices, des crimes, des maux de toute espèce qui sont sortis de la caverne du Directoire renversé, comme d'une autre boëte de Pandore; comptez, si vous le pouvez, le nombre de familles qu'ils ont plongées dans la misère, divisées, décimées ou anéanties; mesurez, s'il vous est possible, les larmes et le sang qu'ils ont fait verser! Le sang de plusieurs millions d'hommes, les larmes de presque tous les peuples des deux hémisphères, condensés sur leurs têtes sacrilèges, $y$ forme un nuage noir, sombre, épais, d'où partira tôt ou 
tard le tonnerre qui doit les écraser. Ombres illustres des victimes de Vendôme, immolées par Viellard sur l'autel des dieux sanguinaires qui ont souillé le Luxembourg! Ombres révérées des républicains trop confiants massacrés à Grenelle, ombres non moins précieuses des démocrates de la Suisse et de l'Italie ! Et vous, ombres généreuses et immortelles de nos héros, livrés aux besoins de toute espèce, et sacrifiés dans les hospices et les combats, pour satisfaire leur soif inextinguible du sang et des richesses, qui vous plaisez sans doute à voltiger dans ce berceau de la liberté, reprenez pour un moment vos cadavres ensanglantés, rassemblez vos membres épars, sortez de vos tombeaux, levez-vous, et venez avec nous, avec vos camarades mutilés, avec vos veuves et vos orphelins, vos pères, vos mères, vos sœurs et vos frères en deuil ! Venez, venez demander avec nous au Corps Législatif une justice entière, une prompte vengeance ! ${ }^{38}$ »

Cet appel à la vengeance est-elle le souhait d'un retour pur et simple à la période de la Terreur? Pour que le culte de la mémoire des martyrs remplisse sa fonction, il faut savoir regarder le passé en face. L'œuvre du gouvernement révolutionnaire est revendiquée, apparemment sans réserves: "la Terreur fut légitime, elle fut nécessaire... ${ }^{39}$.

Quant au terme «jacobin », brandi comme un épouvantail par les directorialistes, il faut savoir le revendiquer, à l'image de ces anciens Hollandais retournant contre les Espagnols le terme de "gueux» : "Nous rattacherons au nom de jacobin, à ce nom, la terreur des rois, de leurs agents et de leurs amis, des traitres et des fripons ; à ce nom conséquemment proscrit et abhorré de tout être vil, méchant, corrompu ou ambitieux ; à ce nom, qui vivra avec honneur dans la mémoire des démocrates de tous les siècles et de tous les pays, la considération qu'il obtint du peuple français dans les beaux jours de la Révolution. » ${ }^{40}$

41 En dépit de ce retour sur le passé, ce discours ne peut pourtant être interprété comme une complainte nostalgique, voire comme une provocation. Ces rappels historiques, cette érection d'une mémoire comme marqueur d'identité collective, sont également destinés à conjurer deux types de comportements symptomatiques d'un peuple qui entre en révolution; l'impatience et l'enthousiasme. Répéter ces comportements en une période où prime la recherche de la stabilité constitutionnelle ne pourrait conduire qu'à une série d'échecs dramatiques pour l'avenir du mouvement démocratique.

C'est pourquoi les rappels du passé ne sont pas exclusivement des faits positifs. Les républicains sont les premiers à pouvoir se plaindre de la période de la Terreur, période d'» aveuglement qui les fit se diviser et s'entr'égorger par l'échafaud " ${ }^{41}$. L'orateur met en garde son auditoire contre les comportements impulsifs :

«Il est bon, il est utile sans doute de bien présumer de ses moyens et de ses ressources ; mais cette présomption louable, sans laquelle on ne saurait prétendre à la victoire dans les combats comme en politique, a ses bornes, si on les dépasse, elle n'est plus que témérité, bravade impuissante, qu'une ridicule fanfaronnade qui convertit en cyprès les lauriers qu'on avait la certitude de cueillir, si on eût écouté la voix modeste de la sagesse et de la prudence... » ${ }^{42}$

Bach s'attache également à dissocier la référence au jacobinisme et le souvenir de la guillotine. Il ne souhaite pas la peine de mort pour les directeurs déchus, mais des peines empreintes d'un souci de pédagogie sociale ${ }^{43}$ :

«Oui, quelque coupables que vous soyez, Reubell, Merlin, et vous tous législateurs, directeurs ou ministres, qui pouvez être leurs complices! Je ne désire pas votre mort, mais que vous soyez condamnés à balayer les rues de Paris, revêtus de ce grand costume qui vous a donné l'orgueil, l'avarice et la cruauté des rois que vous vouliez singer. ${ }^{44}$ 
44 accompagnée d'un programme de « régénération » républicaine : chaque fonctionnaire public, chaque fournisseur de l'État, tous ceux qui exercent des charges publiques, doivent rendre des comptes périodiquement pour garantir la transparence de leurs revenus, ou du moins des revenus qu'ils tirent de leurs fonctions au sein de la République, ou des transactions qu'ils passent avec elle (point 3 du programme de Bach). Cette opération de «moralisation » de la vie publique, inspirée de la maxime de la pensée politique classique, selon laquelle la vertu est le ressort des régimes républicains, est une exigence que l'on retrouve dans les centaines d'adresses expédiées au Corps législatif après les journées de prairial ${ }^{47}$. Elle peut se résumer par un mot d'ordre : «Il faut que la République soit gouvernée par des républicains». Bach fait écho à cette exigence dans son dernier point : « relever l'esprit public en accordant aux républicains la préférence qui leur est due pour tous les emplois civils et militaires ${ }^{48}$... ». Cette " régénération » est également sobrement synthétisée par Le Peletier dans le premier point de son exposé: «Rétablir dans le gouvernement l'esprit démocratique. »

49 D’une façon générale, disponibles en l'état, les mesures proposées par Bach sont plus précises que celles de Le Peletier. Ainsi développe-t-il tout un programme d'économies publiques par la réduction des indemnités parlementaires, ou évoque-t-il la nécessité 
de réhabiliter les « rentiers primitifs" ruinés par la banqueroute des deux tiers. Ces préoccupations ne sont pas propres à Victor Bach, et ne sont pas du tout secondaires pour les démocrates de l'an VII. Nous les retrouvons chez deux autres publicistes de la période, qui sont également deux personnalités importantes du mouvement néojacobin de la fin du Directoire. Bernard Metge, pour établir la confiance entre les petits rentiers et le régime républicain, avait développé le plan d'un établissement de crédit public, liant croissance économique et drainage de l'épargne populaire, totalement soustrait à la tutelle étatique ${ }^{49}$. François Dubreuil, chirurgien-dentiste, a placé parmi les premiers objectifs de la démocratie représentative l'indemnisation des petits détenteurs de rentes d'État ruinés par la politique financière du Directoire ${ }^{50}$.

Victor Bach, pour sa part, insiste sur la nécessité d'établir la progressivité de l'impôt: « D'établir de suite l'impôt progressif, en faisant tourner l'excédent de ce que les riches payeraient par ce moyen, à l'allégement des impôts de la classe industrieuse et laborieuse de la nation ${ }^{51}$. Il préconise un contrôle public sur les hauts revenus (point 6), et limite la contribution « de la classe industrieuse et laborieuse de la nation » aux «trois journées de travail exigées par la constitution pour être admis au nombre des citoyens ». Remarquons au passage que l'orateur s'accommode parfaitement du cadre censitaire de la Constitution de 1795. Concession tactique? En ce cas, ce n'est pas simple opportunité, puisque la brochure de l'année précédente se terminait déjà par un appel à se rallier à la Constitution de l'an III et à respecter la représentation nationale ${ }^{52}$. Quant à tester la sincérité de cette concession, c'est une autre affaire, d'autant plus que se réclamer publiquement de la Constitution de 1793 expose toujours à la peine capitale.

51 Le versement d'une contribution, somme toute très modique pour l'accès aux assemblées primaires, ne constitue pas un enjeu démocratique ${ }^{53}$. En revanche, les transferts obtenus par la progressivité de l'impôt permettraient le financement de deux types de programmes sociaux : un programme d'éducation (repris du plan d'éducation de Michel Le Peletier, nommément cité au point 11$){ }^{54}$ et des projets hérités de la bienfaisance nationale: secours publics à domicile («De procurer aux citoyens infortunés que les maladies et le besoin forcent à se retirer dans les hospices, tous les secours que l'humanité réclame et de s'occuper des moyens de les leur fournir, le plutôt possible, à domicile... ») ${ }^{55}$, plan d'extinction de la mendicité (« D'extirper la mendicité en ouvrant des ateliers de bienfaisance où l'on puisse occuper utilement les enfants, les vieillards, les infirmes, et les ouvriers valides qui seraient momentanément privés d'ouvrage... ») ${ }^{56}$, assistance aux défenseurs de la patrie («D'honorer les défenseurs de la patrie en invitant à toutes les fêtes publiques leurs camarades mutilés, et les plaçant à côté des magistrats du peuple; de pourvoir religieusement et abondamment à tous leurs besoins, d'acquitter exactement les pensions des veuves, des pères, mères et enfants de ceux qui sont morts pour elle, et de ceux qui ont été mutilés dans les combats $»)^{57}$.

52 La tonalité «sociale » du programme de Bach est-elle pour autant une particularité ? Sous une forme plus ramassée, on retrouve ces différents points dans le programme Le Peletier du 18 thermidor: "Établir une éducation égale et commune; ouvrir des ateliers publics pour détruire la mendicité ». Si Bach se montre sensible à la garantie du droit d'asile (« D'offrir un asyle et des secours aux républicains étrangers qui sont ou seront forcés de s'expatrier, et de leur promettre de ne jamais nous immiscer dans la constitution républicaine, qu'ils pourraient se donner, en rentrant dans leurs foyers ») 
${ }^{58}$. On peut toutefois rappeler que la revendication « fédéraliste» de Le Peletier (« faire une fédération générale ») recouvre chez les néo-jacobins un projet interne de remobilisation des gardes nationales, et un projet externe de confédération de républiques démocratiques et souveraines ${ }^{59}$.

Ainsi, les deux programmes diffèrent peu dans leur contenu. L'inscription de l'impôt progressif comme moyen de redistribution des richesses est au cœur du projet néojacobin, que Isser Woloch nomme la "persuasion démocratique» ${ }^{60}$. Chez Bach, la réhabilitation $\mathrm{du}$ crédit public est indissociable du plan de "régénération » républicaine, la confiance forme un tout. Mais la confiance ne se limite pas, comme chez les architectes de la Constitution de 1795, au groupe des propriétaires. La redistribution de la « richesse superflue » au moyen de la progressivité de l'impôt doit permettre d'enraciner d'un même mouvement, et la République et la propriété, et donc dépasser la contradiction soulignée par Paine dans ses critiques de la Constitution de $1795^{61}$.

54 L'insistance mise sur l'impôt progressif par Victor Bach est une différence de taille avec les mesures proposées par Félix Le Peletier; de fait, le programme du premier apparaît beaucoup plus cohérent, tandis que le second semble se limiter à exposer un catalogue. Cela tient, pour le coup, à des considérations tactiques ; le public visé n'est pas le même. Laisser entrevoir que la clef de voûte du plan de défense et de «régénération » de la République, c'est le principe de progressivité fiscale (principe permanent et non mesure temporaire comme peuvent l'être les divers emprunts forcés), voilà qui provoquerait de fortes résistances dans ce groupe de notables républicains avec lesquels l'état-major du mouvement démocrate cherche alors à conclure une alliance stratégique.

Il reste le point le plus controversé du programme de Bach, le dixième point: « D'examiner, si dans un moment où tous les citoyens doivent prendre les armes pour la défense du territoire de la république, il n'est pas juste de les en reconnaître tous copropriétaires avec les citoyens plus fortunés auxquels l'intérêt général, les lois et la Constitution en garantissent la jouissance et l'usufruit pour eux et leurs héritiers; et s'il n'est pas juste en conséquence de proclamer que tous les citoyens auxquels il ne manque d'autre condition, pour être éligibles aux diverses fonctions publiques, que celle d'être propriétaires fonciers, sont susceptibles d'y être appelés. » ${ }^{62}$

Cette revendication, aussitôt brandie par les conservateurs comme un épouvantail de la loi agraire, est en fait un héritage de la bienfaisance nationale définie en l'an II ${ }^{63}$. On ne peut guère plus la ranger parmi les mesures d'inspiration babouviste. Nulle trace d'abolition de la propriété privée, ni de mise en commun des biens. La référence à la "Grande Commune» ou copropriété des citoyens est le rappel d'un principe élémentaire, admis par de larges secteurs de l'opinion publique ${ }^{64}$. Albert Soboul rappelle que Félix Le Peletier soulignait le 20 août 1793 que « le riche soit moins le propriétaire que l'heureux dépositaire d'un excédent de fortune consacré au bonheur de ses concitoyens " ${ }^{65}$. Et, quand le même Félix évoque sobrement dans son programme d'août 1799 la nécessité de "donner des propriétés aux défenseurs de la patrie », il ne demande pas quelque chose de profondément différent de ce que demande Bach. D'ailleurs, Gérard Walter fait remarquer avec raison que la finalité profonde de ce fameux dixième point n'est pas sociale, mais politique: «...de ce principe [la copropriété] il ne tirait d'ailleurs qu'une conséquence strictement politique, notamment l'extension de l'éligibilité aux diverses fonctions publiques à tous ceux qui 
ne pouvaient pas remplir les conditions relatives au minimum de propriété foncière exigé par la loi » ${ }^{66}$. C'est anticiper simplement la querelle sur les « capacités » qui se développe sous la Restauration. Mais, replacée à l'époque du Directoire, la question de l'ouverture des restrictions censitaires au deuxième degré, était assurément sensible. L'élection directe des représentants, sans l'énorme obstacle du second degré, amorce bel et bien un retour à la Constitution de l'an I. N'est-ce pas là ce que l'on redoute vraiment, par-delà la prétendue loi agraire?

Même si Bach ne fut pas le seul à considérer que la "véritable démocratie " résidait dans l'abaissement des conditions censitaires qui séparaient les électeurs des simples citoyens « actifs » ${ }^{67}$ (voire dans leur suppression pure et simple), même si nombre de municipalités se montraient peu regardantes sur cette barrière censitaire, le groupe dirigeant des néo-jacobins de l'an VII comprit bien vite que cette revendication était le talon d'Achille qui fournirait aux conservateurs le prétexte voulu pour déclarer l'inconstitutionnalité de la Réunion du Manège.

Le discours de Bach n'est pas pour autant une provocation. Rien ne laisse supposer qu'il y eut divorce entre un courant " radical » et un courant plus " opportuniste », même si la tonalité des discours, du fait des circonstances, n'est pas la même. Suivant son impulsion, Bach veut répliquer à ses accusateurs, et il prépare un "second discours", qui ne sera jamais prononcé ; un « ami estimable » l'en dissuade, car il pourrait « nuire à la Société ", c'est-à-dire fournir le prétexte à la fermeture du Club. Plusieurs de ses biographes soulignent que ce second discours était bien plus modéré que le premier. N'ayant pu retrouver ce texte, il faut nous en tenir à la constatation de la concertation permanente entre les différentes sensibilités du mouvement néo-jacobin.

Pour mieux replacer Victor Bach au sein de ces différents réseaux, il faut abandonner les quelques traces que nous avons de son action, pour esquisser des rapprochements avec d'autres personnalités.

Par ses idées, tout d'abord, et plus particulièrement par son intérêt pour la fiscalité progressive et le crédit public, il y a des affinités avec ces deux autres publicistes démocrates - d'ailleurs liés entre eux - que sont Bernard Metge et François Dubreuil. Le premier mène une vigoureuse campagne contre Sieyès. Il est l'inspirateur d'une pétition tendant à faire invalider son élection au poste de directeur le printemps précédent ${ }^{68}$; au début du Consulat, il rédige plusieurs pamphlets contre Bonaparte. Dubreuil a pris ce dernier pour cible dès le lendemain du coup d'État de Brumaire; cette réaction rapide invalide l'idée couramment admise qu'il n'y eut pratiquement aucune réaction républicaine au coup de Brumaire ${ }^{69}$. Les listes de proscription du 20 brumaire plaçaient Metge et Dubreuil en bonne place, aux côtés de Bach («Basch») et de Félix Le Peletier.

61 Par la thématique générale, et d'abord par l'attention qu'il porte à la prise en charge par l'autorité publique de la question du paupérisme, Bach est très proche des articles du journal Le Démocrate ou le défenseur des principes, animé par René-François Bescher et Rigomer Bazin, établi rue du Fouarre, en bas du Quartier Latin, en des lieux que Bach connaissait très bien. On trouve encore, au sein de ce groupe, l'imprimeur Lamberthé, Lagrelet, le traiteur Cardinaux, Marchand... Plusieurs connaîtront une destinée tragique, en raison de l'importance qu'ils avaient acquise dans l'organisation d'une opposition démocratique au régime du Directoire, puis du Consulat.

Si le discours de Bach du 30 messidor a pu alimenter une campagne qui aboutit à la fermeture de la Salle du manège, le texte inséré dans Le démocrate intitulé « Réponse à 
Sieyès » fournit des arguments aux mêmes, qui obtiennent la fermeture du club de la rue du Bac le 27 thermidor. Ne reconnait-on pas Sieyès dans ce "prêtre fourbe, astucieux et méchant $"{ }^{70}$ que Bach interpelle ? Ou bien est-ce Talleyrand, qui est plutôt la cible du Journal des hommes libres par les plumes de Charles Hesse, Jorry, Antonelle?

Hors les temps forts de la séquence 1798-1799, le parcours de Bach devient évanescent, vers l'amont comme vers l'aval. Nous avons souligné à plusieurs reprises que ses propositions sociales étaient largement héritées du courant « philanthropique » qui se développa dans les années 1790-1791 autour du Cercle Social. Or, dans les rapports de police, nous trouvons, aux côtés de Victor Bach, un personnage qui joua un rôle très important au début de la décennie révolutionnaire; il s'agit de l'officier de santé Montain-Lambin ${ }^{71}$. Ils sont tous deux mis en arrestation, avec quatre autres militants de la section Chalier, en prairial an III ${ }^{72}$. Il est évident que, dans sa thématique, et souvent dans son personnel, il y a une continuité totale entre les milieux du Cercle Social et les milieux de l'opposition démocratique sous le Directoire.

La personnalité de Victor Bach est beaucoup plus complexe que les clichés réducteurs qui le présentent comme un «énergumène » ou un provocateur. Elle ne correspond pas non plus à la tête du jeune homme foudroyé, mais non défiguré, du tableau de Le Cœur. Elle est énigmatique par bien des aspects, comme le sont encore largement les milieux démocratiques parisiens.

Ce ne sont pas des conspirateurs à part entière, et c'est bien ce qui va causer leur perte. Ils croient à la persistance d'un espace public ouvert par la Révolution, espace de citoyenneté au sein duquel la distance s'amenuise entre dirigeants et dirigés. Ils n'ont pas voulu voir que, si la Constitution de l'an III avait balisé le forum sans en interdire l'accès, le Consulat met un terme à cette proximité volontiers frondeuse. Dubreuil apostrophe durement et immédiatement le nouveau maître, puis disparaît, tandis que Metge cherche par ses libelles à en détruire l'image, ce qui lui vaudra le peloton d'exécution.

Et Victor Bach? Aurait-il été plus lucide que ses frères et amis, et pris au pied de la lettre la devise "Vivre libre ou mourir " ? L'hypothèse est édifiante, mais dérangeante dans la mesure où elle rate son effet : la mort " héroïque » ou " glorieuse ", revendiquée comme telle, doit être publique. Or, tout laisse à penser que si une personnalité ayant la notoriété de Bach en 1799 s'était brûlée la cervelle place de la Concorde, l'événement aurait laissé des traces dans une presse qui n'était pas encore muselée.

$\mathrm{Au}$ terme de cette enquête, je n'ai trouvé ni le cadavre, ni le mobile de l'acte. J'avance trois hypothèses.

68 À coup sûr, il n'est pas mort place de la Concorde. Reste le bois de Boulogne, comme l'indique la police. Or, ce lieu est celui des duels.

Le Journal des Hommes libres nous apprend qu'en prairial an VIII des altercations ayant dégénéré en duel opposaient des démocrates, les uns partisans du ralliement à Sieyès, les autres hostiles. Le grand adversaire des jacobins au cours de l'été précédent, cherchait maintenant à fédérer l'opposition à Bonaparte ; les préoccupations tactiques ont dû être bien mal acceptées, cette fois, surtout dans les milieux qui s'efforçaient, il y a peu, de mettre hors la loi «l'obscur métaphysicien». Bach aurait-il été de ces intransigeants? C'est plausible.

Ou bien, s'il y a eu provocation policière, un agent a pu chercher à éliminer une personnalité gênante, et faire d'une pierre deux coups ; disparaît ainsi un adversaire de 
longue date, et un démocrate à principes, trop intransigeant. On sait que Fouché était du complot contre le Premier Consul à cette époque.

71 Reste la dernière hypothèse ; Bach s'est vraiment tiré une balle dans la tête, à la nouvelle de la victoire de Marengo, qui ruine les espérances de l'opposition, et consolide le pouvoir de Bonaparte. Mais le cadavre disparait sans laisser de traces, sans passer par la basse geôle, qui n'enregistrait de toutes façons que les suicidés anonymes. Ses amis l'ont-ils emporté pour l'enterrer discrètement? Ou bien est-ce une police désormais mieux rôdée qu'elle ne l'était en brumaire, qui s'est chargée de la besogne, effaçant un ultime témoignage public de protestation?

Quoi qu'il en soit, le « cas » Bach nous invite à une nouvelle appréciation positive des réseaux démocratiques sous le Directoire et le Consulat. Le tableau de Le Cœur, qui inaugure cette enquête, est une représentation de la connaissance historique à un moment donné. Les déplacements qui se sont opérés dans le champ de connaissance de la période finale du Directoire et de Brumaire, ouvrent la voie à d'autres configurations narratives.

\section{NOTES}

1.Jean-Paul BERTAUD, Bonaparte prend le pouvoir, Éditions Complexe, 1987 ; Jean TULARD, Napoléon ou le mythe du sauveur, Paris, Fayard, 1986 ; Alphonse AULARD, « Les lendemains de Brumaire ", in Études et leçons sur la Révolution française, Paris, Félix Alcan, 2e série, 1902, pp. 213-252 ; Pierre SERNA, « Refaire l'Histoire, écrire l'Histoire, ou comment raconter le 18 Brumaire entre 1800 et 1802 ?", intervention au colloque de Potenza (mai 1999) : La parabolla della democrazia (actes à paraître).

2.Michel VOVELLE, La Révolution française. Images et récits, tome V, pp.334-341.

3.François воUснот, Le général Bonaparte au Conseil des Cinq-Cents, à Saint-Cloud, le 10 novembre 1799, 1840, Musée national du château de Versailles.

4.Vive la République! (1792-1992), Catalogue de l'exposition qui s'est tenue aux Archives nationales, Hôtel de Rohan, de septembre 1992 à janvier 1993, p. 39.

5.Tous les renseignements disponibles sur J.Le Cœur, c'est-à-dire très peu de choses, sinon que le tableau a été donné en 1895 au musée Carnavalet par le préfet Poubelle, m'ont été aimablement communiqués par M.Jean-Marie Bruson, conservateur. Il souligne notamment qu'il ne faut pas confondre ce Le Cœur, et son homonyme beaucoup plus connu, qui se prénommait Jean-Baptiste, mort en 1838 !

6.Biographie nouvelle des contemporains ou Dictionnaire historique et raisonné de tous les hommes qui, depuis la Révolution, ont acquis la célébrité, sous la direction de Arnault et Jay (1820-1825).

7.Il s'agit bien entendu de la loi du 22 floréal an VI (11 mai 1798) dirigée contre les néojacobins. La chronologie directoriale reste bien mal fixée ; à propos de la même loi, la biographie Didot (cf. infra) lui attribue la date du 23 février an VII, Michaud (cf. infra) place les élections de l'an VI en 1799, etc. 
8.Autre erreur : le discours de Bach a été prononcé à la tribune du Club du Manège, la salle étant située sur le pourtour du jardin des Tuileries.

9.Biographie universelle ancienne et moderne.

10.Supplément à la Biographie universelle, tome 57, p. 9.

11.Nouvelle Biographie Universelle, chez Firmin Didot, tome 3, 1852, p. 53.

12.Dictionnaire de Biographie française, sous la direction de PRÉVOST et ROMAN D'AMAT, tome IV, 1948, pp. 1045-1046.

13.La grande conspiration anarchique de l'Oratoire, renvoyée à ses auteurs, par le citoyen Bach, médecin de la ci-devant Université de Montpellier, 7 p., s.d., Bibliothèque historique de la Ville de Paris, 604481. Premier discours du citoyen Bach, médecin, électeur à l'Oratoire en l'an 6, prononcé à la Réunion du Manège, le 30messidor an VII, 36 p. (imprimerie Benoist, messidor an VII), Bibliothèque nationale de France, Lb40 2358.

14.Docteur Raymond LAURIÈRE, « Les médecins du Bas-Rouergue et la Révolution », in numéro spécial du Bulletin de la Société des Amis de Villefranche-de-Rouergue (1989-1990), «La Révolution et le Rouergue », pp. 161-176. Cet article reproduit pour l'essentiel les informations contenues dans un article de A.COIFFARD, « Victor Bach, médecin et homme politique », in Nouvelles Chroniques villefranchoises, première série, Salingardes, Villefranche-de-Rouergue (1945). Plusieurs orthographes sont possibles dans les sources concernant le personnage : Bach, ou Bache, ou Basch. Nous avons retenu Bach parce que c'est celle des imprimés, même si la prononciation devait être « Bache ", à la française, plutôt qu'à l'allemande.

15.Jacques HILlaiRet, Connaissance du Vieux-Paris, Éditions de Minuit, 1951, tome 1, p. 270. Le Journal des hommes libres, $\mathrm{n}^{\circ} \mathrm{du} 18$ fructidor an VIII, évoque ladite statue comme un « vieux plâtre », qui aurait été déplacée de la place de la Concorde parce qu'elle gênait la vue, pour être déposée sur un tertre à la périphérie du Champ-de-Mars. 16.Richard СОВB, La mort est dans Paris : enquête sur le suicide, le meurtre et autres morts subites à Paris au lendemain de la Terreur, Éd. Le Chemin Vert, 1985.

17.Archives de Paris, D4 U1 (7).

18. Le Moniteur Universel, numéro du 6 frimaire an VIII (27 novembre 1799)

19.Archives de la Préfecture de police de Paris, Aa/274, pièce $n^{\circ} 7$. À notre connaissance, la rubrique que Raymonde MONNIER consacre à Victor « Bache" (Répertoire du personnel sectionnaire parisien en l'an II, Publications de la Sorbonne, 1985, pp.

488-489), est la seule qui mentionne le bois de Boulogne comme lieu du suicide ; mais tout en le maintenant dans le contexte de brumaire, il est vrai (« décrété d'arrestation le 20 brumaire an VIII. Il s'est suicidé d'un coup de pistolet au bois de Boulogne, peu après.").

20.Archives de Paris, D4 U1 (7).

21.Alphonse AULARD, «Les derniers jacobins ", La Révolution française, tome 26, 1894, pp. 80-94, interprète le programme présenté par Bach comme un " programme néobabouviste ", prônant le retour à l'an II et le "socialisme". Mais il en souligne toute l'importance : "Cette apologie de la république démocratique eut un grand retentissement dans le pays, et scandalisa l'opinion moyenne."

22.Bibliothèque nationale de France, manuscrit $n^{\circ} 2718$ : «Assemblée de l'Oratoire, 27 germinal an VI".

23.Isser Woloch, Jacobin Legacy, Princeton, 1970, pp. 311-332 ; Jean-René SURATTEAU, Les élections de l'an VI et le coup d'État du 22 floréal (11 mai 1798), Paris, Belles-Lettres, 1971, pp. 228-248. 
24.Titre IV, article 35, Jacques GODECHOT, Les Constitutions de la France depuis 1789, Garnier-Flammarion, 1979, p. 107.

25.Raymond LAURIÈRE, « Les médecins du Bas-Rouergue et la Révolution », op. cit.

26. Raymonde MONNIER, Répertoire du personnel sectionnaire, op. cit., p. 448 ; AN F7 4588, plaquette $n^{\circ} 3$ : dossier Bach et autres, du 2 prairial an III (21 mai 1795).

27.Albert SOBOUL, Les sans-culottes parisiens en l'an II : mouvement populaire et gouvernement révolutionnaire ; 2 juin 1793-9 thermidor an II, Paris, Librairie Clavreuil, 1962, p. 418.

28.André MARTIN et Gérard WALTER, Catalogue de l'histoire de la Révolution française, Département des imprimés de la Bibliothèque nationale de France, tome 1, 1936 ; rubrique «Bach".

29.Pièce adjointe à La grande conspiration anarchique..., op. cit.On y apprend que son loyer est de 40livres par terme, ce qui porte de nouveau les revenus imposés de Bach (167 livres) au-dessus du seuil censitaire requis par la Constitution (cent cinquante journées de travail).

30.Premier discours..., op. cit., p. 34. Cet intermédiaire s'appelle Ricome.

31.Ami des lois, $\mathrm{n}^{\circ} 1423$ du 3 thermidor an VII (21 juillet 1799) : Bach aurait «proposé le partage des terres et mille autres folies semblables".

32.Pierre SERNA, Antonelle, aristocrate révolutionnaire; 1747-1847, Éditions du Félin, 1997, pp.374-388 ; Laurence conSTANT, Félix Le Peletier de Saint-Fargeau. Un itinéraire de la Révolution à la monarchie de Juillet, Découvrir, jeunes talents, 1995, pp. 82-85.

33. Françoise BRUNEL et Sylvain GOUJON, Les martyrs de prairial, Éditions Georg, Genève, 1992, pp.35-40.

34.Premier discours, op. cit., pp. 4, 8, 16 notamment.

35.Id., p. 8.

36. Robert LEGRAND, Babeuf et ses amis, Publication de la Société des études robespierristes, Paris, 1981, p. 290. L'auteur signale également l'invocation de Marchand, à la tribune de la Société de la rue du Bac, le 16 thermidor an VII. Sylvain Goujon (Les martyrs de prairial, op. cit., p. 77) ne mentionne que ce discours de Marchand. 37.Isser WOLOCH, Jacobin Legacy, op. cit., pp. 46-47.

38.Premier discours, op. cit., pp. 13-14. Cette évocation de la foule des morts qui se relève pour réclamer réparation est profondément inscrite dans l'imaginaire collectif ; la scène se retrouve pratiquement à l'identique à la fin du film d'Abel Gance sur la Première Guerre mondiale, J'accuse.

39.Id., p. 7.

40.Id., pp. 20-21.

41.Id., p. 7.

42.Id., p. 8 .

43.La représentation des directeurs et les peines réclamées s'inspirent des nombreux pamphlets antidirectorialistes parus à l'occasion des journées de prairial an VII (Bernard GAINOT, Le mouvement néo-jacobin à la fin du Directoire. Structure et pratiques politiques, thèse soutenue à Paris I, sous la direction de Michel Vovelle, janvier 1993), dont la thématique centrale est celle du « monde à l'envers" (Ouzi ELYADA, « La presse populaire parisienne et le temps de Carnaval, 1788-1791 », in L'image de la Révolution française, Pergamon Press, 1989, tome 1, pp. 109-110).

44.Id., p. 33. 
45.Le Moniteur, numéro du 20 thermidor an VII, compte rendu de la séance du 18 thermidor de la Réunion des Amis de l'Égalité et de la Liberté ; Alphonse AULARD (Les derniers jacobins, op. cit.) présente également un condensé de ce programme, mis au point par la commission d'instruction publique de la Société de la rue du Bac, dont Félix Le Peletier est rapporteur.

46. Christine PEYRARD, «La revendication du droit à l'association ; les débats de l'an VII sur l'association politique », in Les Droits de l'homme et la conquête des libertés; des Lumières aux révolutions de 1848, Vizille, 1986, pp. 311-318.

47.Bernard GAINOT, « Les adresses de félicitations parvenues au Corps législatif après les journées des 29-30 prairial an VII », in Annales historiques de la Révolution française, $\mathrm{n}^{\circ}$ 273, 1988.

48.Premier discours, op. cit., p. 27.

49.Bernard GAINOT, " Un itinéraire démocratique post-thermidorien : Bernard Metge ", in Pour la Révolution française. Hommages à Claude Mazauric, sous la direction de Christine LE BOZEC et Éric WAUTERS, Publications de l'Université de Rouen, 1998, p. 98 (« Projet de banque générale d'agriculture et de commerce, 25 ventôse an VI-15 mars 1798”).

50.Bernard GAINOT, « La notion de démocratie représentative ; le legs néo-jacobin de 1799 », in L'image de la Révolution française, Pergamon Press, 1989, tome 1, pp. 523-529 (analyse de la brochure de Dubreuil, Hommage à la vérité, contre l'oppression, l'injustice, l'inhumanité, et les rapines du Directoire et de ses représentants au Corps législatif, nivôse an VII - décembre 1798).

51.Premier discours, op. cit., p. 23 (point 4 du programme).

52.La grande conspiration anarchique..., op. cit., p. $6:$ « Rallions-nous tous autour de la constitution de l'an 3 : n'oublions pas que, si le dépôt en fut remis à la fidélité de nos premiers magistrats, il fut aussi confié à notre courage". Ce peut être là une autre formulation de la " démocratie représentative".

53.Jean-Pierre GROSS, Fair shares for all. Jacobin egalitarianism in practice, Cambridge University Press, 1997, pp. 122-144 ; Bernard GAINOT, « Pierre Guyomard et la revendication démocratique, dans les débats autour de la Constitution de l'an III ", in 1795 : Pour une république sans révolution, Presses Universitaires de Rennes, 1996, sous la direction de Roger DUPUY et Marcel MORABITO, pp. 261-273.

54.Premier discours, op. cit., p. 25. L'éducation est réservée chez Bach aux garçons : « De s'occuper des moyens de donner à tous les enfants mâles de la République une éducation commune et martiale, ainsi que l'avait proposé l'immortel Michel Le Peletier". L'ultradémocratie a donc ses limites.

55.Id., pp. 25-26. C'est le point 13.

56.Id., p. 26. C'est le point 14.

57.Id., p. 24. C'est le point 9.

58.Id., p. 24. C'est le point 8.

59.Bernard GAINOT, « Révolution, Liberté = Europe des nations ? La sororité conflictuelle », in Sur la Révolution, approches plurielles. Mélanges Michel Vovelle, I.H.R.F., Société des études robespierristes, 1997, pp. 457-458.

60.Isser WOLOCH, Jacobin Legacy, op. cit., pp. 171-186, tout particulièrement.

61. Yannick BOSC, « Thomas Paine et les constitutions de 1793 et 1795 ; critique de la République formelle ", in Thomas Paine ou la République sans frontières, études réunies sous la direction de Bernard VINCENT, 1993, pp. 79-86.

62.Premier discours, op. cit., p. 25. 
63. Catherine DUPRAT, Pour l'amour de l'humanité. Le temps des philanthropes, Éditions du CTHS, Paris, 1993, tome 1, pp. 332-357.

64.Jean-Pierre GROSS, Fair shares..., op.cit., pp. 145-178. Mais il faut rappeler que cette référence à la propriété commune originelle n'est pas propre au jacobinisme.Ainsi, Cabanis le 26 janvier 1799, « La Nation est la Grande Commune. Il ne faut considérer que des propriétés particulières consacrées par le travail ; tout le terrain qu'elles ne couvrent pas est propriété nationale (propriété primordiale) », Opinion sur le partage des communaux, Archives nationales, ADXVIIIa (12).

65.Albert SOBOUL, Les sans-culottes parisiens..., op. cit., p. 467.

66.Dictionnaire de biographie française, op. cit., p. 1046.

67.Bernard GAINOT, « Pierre Guyomard et la revendication démocratique... ", op. cit.

68.AN, C686 ; Avis aux Cinq-Cents d'avoir à vérifier si Siéyès ne pourrait pas être constitutionnellement destitué comme l'a été Treilhard, 27 thermidor an VII (14 août 1799).

69.Dubreuil à Bonaparte, $\mathrm{BNF}, \mathrm{Lb}(43) 31$; daté du 1er frimaire an VIIIde la République, 16 pages.

70.Premier discours..., op. cit., p. 3.

71.Catherine DUPRAT, Le temps des philanthropes, op. cit., p. 276. L'auteur présente Montain-Lambin comme " l'homme de la bienfaisance générale"; Raymonde MONNIER, Répertoire du personnel sectionnaire, p. 491.

72.F7 4588, dossier « Basch et autres... », cf. supra.

\section{RÉSUMÉS}

Dans les années 1880, un certain Le Cœur a peint un tableau intitulé La mort de Victor Bach, le 18 Brumaire, au pied de la statue de la Liberté, place de la Concorde. Ce tableau est en rupture avec toutes les représentations officielles du 18 Brumaire. Il met en scène le suicide d'un médecin démocrate, l'un des porte-parole du mouvement démocratique parisien à la fin du Directoire. Conformément au genre de la peinture d'histoire de cette époque, il s'appuie sur quelques notices biographiques disponibles, susceptibles de fournir une connaissance positive. Or, il s'avère que les faits sont loin d'être définitivement établis; tant pour la matérialité des circonstances de la mort, et même pour la date de celle-ci, que pour la centralité de la référence babouviste, dès lors que l'on aborde la nature de l'opposition démocratique entre 1796 et 1799. La figure de Victor Bach se détache alors de sa reconstruction dramatique, pour mieux nous aider à saisir la complexité des réseaux néojacobins, et les enjeux politiques de la période de la fin du Directoire et des débuts du Consulat.

An Inquiry into the "Suicide" of Victor Bach. In the 1880s, a certain Le Cœur painted a picture entitled The Death of Victor Bach on 18 Brumaire at the Foot of the Statue of Liberty on the Place de la Concorde. This picture runs counter to all the official representations of 18 Brumaire. It portrays the suicide of a physician and democrat, who was one of the spokesmen of the democratic movement in Paris at the end of the Directory. In accordance with historical genre painting at this time, its positive assumptions are based on scanty biographical data. But the facts are far from clearly ascertained, whether it be the material circumstances of his death, even the date of its occurrence, or its necessary relevance to Babouvism, in the broader context of the democratic opposition between 1796 and 1799. Thus, the figure of Victor Bach emerges from its 
dramatic reconstruction and can help us pinpoint the complexity of the neo-Jacobin networks and the political issues involved during the period spanning the end of the Directory and the advent of the Consulate.

Inchiesta sul suicidio di Victor Bach. Negli anni 1880 un certo Le Coeur ha dipinto un quadro intitolato La morte di Victor Bach, il 18 Brumaio, ai piedi della statua della libertà, piazza della Concordia. Questo quadro rappresenta una frattura con tutte le rappresentazioni ufficiali del 18 Brumaio. Esso mette in scena il suicidio di un medico democratico, uno dei portavoce del movimento democratico parigino alla fine del Direttorio. Confermemente al genere della pittura di argmento storico di quell'epoca, esso si fonda su qualche notizia biografica disponibile, in grado di fornire una conoscenza positiva. Ora, si verifica che i fatti sono lungi dall'essere definitivamente stabiliti, tanto per la materialità delle circostanze della morte, e anche per la data di es sa, quanto per la centralità del riferimento babuvista, non appena si affronta la natura dell'opposizione democratica tra 1796 e 1799. La figura di Victor Bach si stacca allora dalla sua ricostruzione drammatica, per meglio aiutarci a cogliere la complessità delle reti giacobine e la posta politica in gioco nel periodo della fine del Direttorio e degli inizi del Consolato.

En los años 1880, un tal Le Couer pintó un cuadro titulado: La mort de Victor Bach, le 18 Brumaire, au pied de la statue de la Liberté, place de la Concorde. Este cuadro rompe con las representaciones oficiales del 18 Brumaire. Representa un médico democrata, uno de los portavozes del movimiento democrático parisiano al final del Directorio. Los testigos de los actores, spectatores o víctimas de Brumaire, contan quizás otra historia, menos mítica, más humana, que intenta demostrar que si el golpe no fue irresistible, nadie estaba puesto a resistir. La fascinación que ejerció Bonaparte, incluso sobre sus adversarios, superan la irritación provocada por las ambiciones queya se le suponía, borrando las torpezas del 19 brumaire y explicando sin duda en parte las adhesiones que siguen.

Untersuchung über Victor Bachs "Selbstmord". In den 80er Jahren des 19. Jahrhunderts hat ein gewisser Le Cœur ein Bild gemalt, das Victor Bachs Tod am 18. Brumaire, am Fuß der Freiheitsstatue auf der Place de la Concorde heißt. Dieses Gemälde bricht mit allen offiziellen Darstellungen des 18. Brumaire. Es zeigt den Selbstmord eines demokratischen Arztes, eines der Wortführer der Demokraten am Ende des Direktoriums. Der Gattung der historischen Malerei dieser Zeit gemäß stützt es sich auf einige biographische Notizen, die geeignet waren, eine positive Kenntnis der Tatsachen zu verschaffen. Es erweist sich aber, daß das alles nicht so einwandfrei feststeht. Dies betrifft ebenso die Umstände und sogar das Datum des Todes wie die unmittelbare Referenz an Baboeuf, die fraglich wird, sobald man die Beschaffenheit der demokratischen Opposition zwischen 1796 und 1799 betrachtet. Die Figur des Victor Bach hebt sich nun aus ihrer demokratischen Rekonstruktion hervor und hilft uns, die Komplexität der neo-jakobinistischen Verbindungsnetze sowie die politischen Einsätze am Ende des Direktoriums und am Anfang des Konsulats besser zu verstehen.

\section{AUTEUR}

\section{BERNARD GAINOT}

Université de Paris I - I.H.R.F. 